\title{
Application of Lidocaine Spray for Tracheal Intubation in Neonates - A Clini- cal Trial Study
}

\author{
Mirhadi Mussavi ${ }^{1}$; Khairollah Asadollahi ${ }^{2,3, *}$; Ghobad Abangah ${ }^{4}$; Sirus Saradar ${ }^{1}$; Naser Ab- \\ basi $^{5}$; Fereidon Zanjani ${ }^{6}$; Mahsa Aminizade ${ }^{7}$ \\ ${ }^{1}$ Department of Pediatrics,Pediatric research center, Faculty of Medicine, Tabriz University of Medical Sciences, Tabriz, IR Iran \\ ${ }^{2}$ Department of Epidemiology, Faculty of Medicine, Ilam University of Medical Sciences, Ilam, IR Iran \\ ${ }^{3}$ Research Centre for Psychosocial Injuries, Ilam University of Medical Sciences, Ilam, IR Iran \\ ${ }_{5}^{4}$ Department of Gastroenterology, Ilam University of Medical Sciences, Ilam, IR Iran \\ ${ }_{6}^{5}$ Department of Pharmacology, Iran University of Medical Sciences, Tehran, IR Iran \\ 6 Department of Anaesthesiology, Faculty of Medicine, Tabriz University of Medical Sciences, Tabriz, IR Iran \\ ${ }^{7}$ Department of Emergency Medicine, Faculty of Medicine, Tabriz University of Medical Sciences, Tabriz, IR Iran \\ ${ }^{*}$ Corresponding author: Khairollah Asadollahi, Department of Epidemiology, Faculty of Medicine, Ilam University of Medical Sciences, Ilam, IR Iran. Tel: +98-8412227126, Fax: +98- \\ 8412227120, E-mail: masoud_1241@yahoo.co.uk \\ Received: February 21, 2014; Accepted: July 15, 2014
}

\begin{abstract}
Background:Tracheal intubation is extremely distressing, painful, and may influence heart rate and blood pressure. Sedatives, analgesics, and muscle relaxants are not commonly used for intubation in neonates.

Objectives: This study aimed to evaluate the effects of lidocaine spray as a non-intravenous drug before neonatal intubation on blood pressure, heart rate, oxygen saturation and time of intubation.

Patients and Methods: In a randomized, controlled study each neonate was randomly assigned to one of the two study groups by staffs who were not involved in the infant's care. The allocation concealment was kept in an opaque sealed envelope, and the investigators, the patient care team, and the assessors were blinded to the treatment allocation. The selected setting was NICU unit of a teaching hospital in Ilam city, Iran and participants were 60 neonates with indication of tracheal intubation with gestational age $>30$ weeks. Patients in the treatment group received lidocaine spray and the placebo group received spray of normal saline prior to intubation. Main outcome measurements were the mean rates of blood pressure, heart rate, oxygen saturation, intubation time and lidocaine side effects were measured before and after intubation.

Results: Totally 60 newborns including 31 boys and 29 girls were entered into the study (drug group $n=30$; placebo group $n=30$ ). Boy/girl ratio in treatment and placebo groups were 1.3 and 0.88 , respectively. Mean age \pm SD of participants was $34.1 \pm 24.8$ hours (treatment: $35.3 \pm$ 25.7; placebo: $32.9 \pm 24.3 ; \mathrm{P}<0.0001$ ). Mean weight \pm SD of neonates was $2012.5 \pm 969 \mathrm{~g}$. Application of lidocaine spray caused a significant reduction of mean intubation time among treatment group compared with placebo group (treatment: $15.03 \pm 2.2$ seconds; placebo: 18.3 \pm 2.3 seconds; $\mathrm{P}<0.0001)$. Mean blood pressure, heart rate and oxygen saturation rate, among neonates in treatment group was reduced after intubation compared with their relevant figures before intubation; however, their differences were not statistically significant except for mean oxygen saturation rate that was reduced significantly in placebo group. No side effects were observed during study.

Conclusions: Though the current study revealed some promising results in the application of lidocaine spray during neonatal intubation without any considerable side effects; however, the current investigation could only be considered as a pilot study for further attempts in different locations with higher sample sizes and in different situations.
\end{abstract}

Keywords: Neonate; Intratracheal Intubation; Lidocaine Spray

\section{Background}

Tracheal intubation is performed frequently in NICU and delivery rooms for neonates with different conditions. This procedure is extremely distressing, painful, and potentially deleterious for airway. Intubations cause many side effects such as pain and stimulation of parasympatic system, hemodynamic changes, raised intracranial pressure and increased risk of intracranial hemorrhage (1-5). There are several ways to reduce pain and vagal stimulation during intubation such as intravenous injection of sedative drugs and neuronal block with lidocaine. Premedication with sedatives, analgesics, and muscle relaxants are standard practices for intubation in pediatrics and adults but they are very limited in neonates (6-12). A consensus statement from the International Evidence-Based Group for Neonatal Pain concluded that "tracheal intubation without the use of analgesia or sedation should be performed only for resuscitation in the delivery room or for life-threatening situations associated with the unavailability of intravenous access (13). Subsequently, in a recent policy statement the American Academy of Pediatrics also recommended that every health care facility caring for neonates implement an ef- 
fective pain-prevention program and use pharmacologic and no pharmacologic therapies for the prevention of pain associated with procedures (14).

Some of the reasons offered for not using premedication before intubation are: concern for adverse reactions and/ or toxic effects of the medications, inadequate time for administration of medications in emergency situation, and the perception that risk/benefit ratios are worsened by using premedications. Also the most frequently used drugs in intubation have considerable complications (15). Lidocaine was used commonly in neonates in circumcision (Topical cream form:cream emla), convulsion and arrhythmia (intravenous form) but retropharyngeal lidocaine spray was not used in neonates for intubation purposes. Lidocaine creates some complications such as seizures, arrhythmia, decreased level of consciousness, and therefore the main issue is the safety of its application in the infant's mucosa. Textbooks of neonatology have not prohibited the application of lidocaine for mucosal purposes; however, some harmful side effects have been attributed to the oral application of this drug (16). If it is shown that the mucosal use does not cause toxic blood levels, this drug can be used in neonatal period more safely. This medicine does not require intravenous access and the use of local rather than intravenous can be used with less complications. Few studies investigated the use of lidocaine at the vocal cord and retropharyngeal areas and reported that plasma levels following topical administration of lidocaine spray (4 $\mathrm{mg} / \mathrm{kg}$ ) reached plasma levels of about $10 \mu \mathrm{g} / \mathrm{mL}$ that is not toxic for pediatric patients $(17,18)$. However, in this study we have used a lower dose of lidocaine.

\section{Objectives}

In this study, we investigated the effects and any side effects of using lidocaine spray on parasympathic stimulation such as blood pressure, heart rate and oxygen saturation (SO2) and time of intubation in neonates.

\section{Patients and Methods}

All neonates with indication of tracheal intubation, with GA $>30$ weeks and without oropharyngeal anomalies, central nervous system (CNS) disorder, congenital heart diseases or arrhythmia admitted to neonatal care unit of Mustafa Khomeini Hospital in Ilam city, in west Iran, between March 2012 and August 2013 were sequentially enrolled in the study. Excluding criteria were neonates less than 30 weeks GA, neonates with renal masses or anomalies, those with oropharyngeal anomalies, congenital heart diseases or arrhythmia, CNS disorders and family history of allergy to corn or porphyria. A randomized, controlled study was performed. Each neonate was randomly assigned to one of the two study groups by staffs who were not involved in the infant's care. Infants were allocated into each group by a randomization method. The allocation concealment was kept in an opaque sealed envelope, and the investigators, the patient care team, and the assessors were blinded to the treatment allocation. The first group was studied with drug A (one puff lidocaine spray, $6.5 \% / \mathrm{dose}, 3 \mathrm{mg} / \mathrm{kg}$ ) and other group with drug B (one puff normal saline spray). Blood pressure, heart rate, and oxygen saturation (SO2) of all neonates were measured electronically via Saadat monitoring instrument (Tossan ind.) and lidocaine side effects (level of consciousness, arrhythmia, and convulsion) were measured by neonatologist before starting the intubation. Thirty seconds before intubation, one puff of drug A or B was sprayed to retropharyngeal and supraglottis of neonates. As soon as the blade of laryngoscope was inserted, duration of intubation was measured with chronometer and 30 seconds later, blood pressure, heart rate and $\mathrm{SO} 2$ were measured again with the same monitor and lidocaine side effect by the same neonatologist and all data were recorded in prepared sheets containing gestational age, age, weight and Apgar score of neonates. During intubation the oxygen flow ( $5 \mathrm{~L} / \mathrm{min}$ ) was maintained for all neonates and no other medications such as sedatives, analgesics were administrated before the study. Spray of normal saline and lidocaine wsa prepared by Pharmacist outside the study and was delivered to the group by the same colors and shapes that were called drug A or B and nobody in the study group knew about drugs contents. Lidocaine or normal saline sprays did not create liquid flow with risk of aspiration at dose of one puff. All intubations were performed by one person (Trained pediatrician). Student $t$ test and paired t test were applied for comparison of the mean rates of quantitative data of different variables in the same group and among 2 groups respectively. Descriptive data were shown by frequency tables or figures.

The study design was approved by the Ethics Committee of Ilam University of Medical Sciences, and informed consent was obtained from the parents of all neonates after explaining the situation to study.

\section{Results}

Totally 60 newborns including 31 boys and 29 girls were entered into the study. Boy/girl ratio in treatment and placebo groups were 1.3 and 0.88 , respectively. Mean age \pm SD of participants was $34.1 \pm 24.8$ hours (treatment: 35.3 \pm 25.7 ; placebo: $32.9 \pm 24.3 ; \mathrm{P}<0.0001$ ). Mean weight and standard deviation of neonates was $2012.5 \pm 969 \mathrm{~g}$ and the differences between mean weight as well as mean age of neonates in treatment group compared with the associated figure in placebo group were statistically significant $(\mathrm{P}<0.0001)$ (Table1). Application of lidocaine spray caused a significant reduction of mean time among treatment group compared with placebo group (treatment: $15.03 \pm$ 2.2 seconds; placebo: $18.3 \pm 2.3$ seconds; $\mathrm{P}<0.0001$ ) (Figure 1). Mean blood pressure, heart rate and oxygen saturation rate, among neonates in treatment group was reduced after intubation compared with their relevant figures before intubation; however, their differences were not statistically significant, except for mean oxygen saturation rate that was reduced significantly in placebo group (Table 2). 
Table 1. Demographic Characteristics of Neonates Participated in the Intubation Study Using Spray of Lidocaine

\begin{tabular}{lccc}
\hline & Treatment & Placebo & Total \\
\hline Gender, $\mathbf{n}$ & & & \\
Male & 17 & 14 & 31 \\
\hline Female & 13 & 16 & 29 \\
\hline Age, $\mathbf{h}$ & $35.3 \pm 25.7$ & $32.9 \pm 24.3$ & $34.1 \pm 24.8$ \\
Weight, $\mathbf{g}$ & $2110 \pm 1077$ & $1915 \pm 854$ & $2012.5 \pm 968.7$ \\
P value & $<0.0001$ & $<0.0001$ & \\
\hline
\end{tabular}

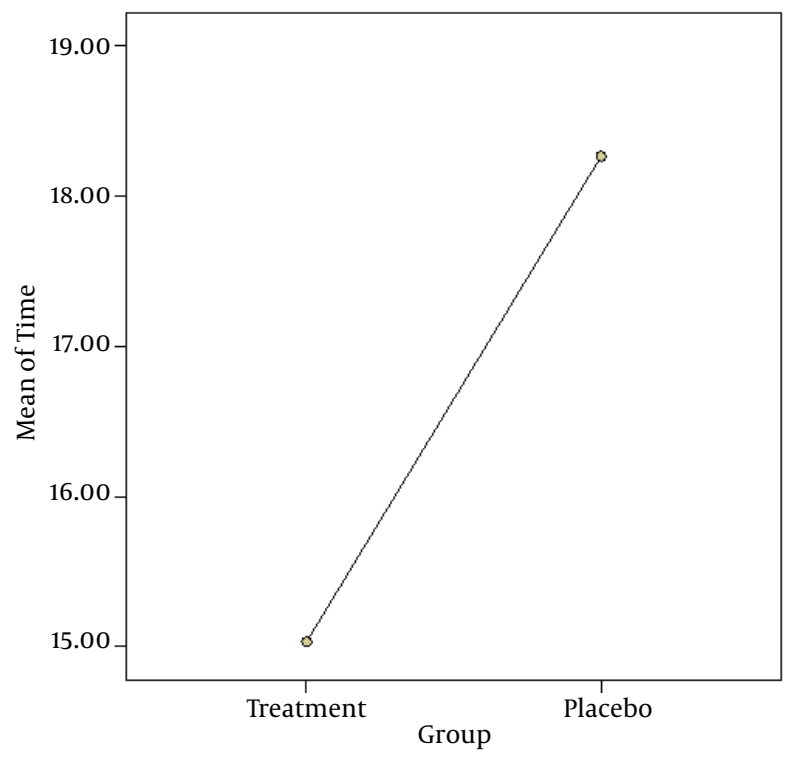

Figure 1. Comparison Between Mean Intubation Time in Treatment and Placebo Group of Neonates

Table 2. Mean and Standard Deviations of Different Studied Variables in Neonates Participated in the Intubation Study Using Spray of Lidocaine ${ }^{\mathrm{a}, \mathrm{b}}$

\begin{tabular}{lcc}
\hline & Treatment & Placebo \\
\hline BP & & \\
\hline BP1 & $71.8 \pm 15.1$ & $71.5 \pm 14.3$ \\
\hline BP2 & $71.4 \pm 15.8$ & $73.8 \pm 13.9$ \\
\hline P value & 0.9 & 0.6 \\
\hline HR & & \\
\hline HR1 & $141.9 \pm 16.5$ & $140.1 \pm 15.8$ \\
\hline HR2 & $139.5 \pm 11.6$ & $139.8 \pm 17.7$ \\
\hline P value & 0.2 & 0.8 \\
\hline OS & & \\
\hline OS1 & $93.7 \pm 3.6$ & $96.1 \pm 2.4$ \\
\hline OS2 & $93.1 \pm 2.3$ & $93.1 \pm 2.5$ \\
\hline P value & 0.3 & 0.0001 \\
\hline Intubation time, sec & $15.03 \pm 2.2$ & $18.3 \pm 2.3$ \\
\hline P value & 0.0001 & 0.0001 \\
\hline a Abbreviations: BP, Blood & Pressure; HR, Heart & Rate; OS, Oxygen \\
Saturation \\
b Data are Presented as Mean \pm SD.
\end{tabular}

\section{Discussion}

Pharyngeal stretching during laryngoscopy triggers sympathetic and parasympathetic reflexes, causing cardiovascular responses. In the vigorous and awake infants, the muscular efforts to resist laryngoscopy and the attempts to cry are accompanied by increases in intrathoracic pressure and reduced venous return may impair venous return from the brain, resulting in intracranial venous hypertension. On the other hand tracheal intubation in alert preterm neonates is accompanied by significant increases in intracranial pressure (ICP), which may contribute to the risk of intraventricular hemorrhage (IVH) $(9,10,18)$. Currently, there is no non-injectable medication to be applied during neonatal intubation, therefore due to some reports on lack of significant side effects of lidocaine spray under blood level of $10 \mu \mathrm{g} / \mathrm{kg}$ in pediatric populations $(19,20)$, and on the other hand, the existence of some reports on harmful side effects of lidocaine (16), we conducted this study to evaluate effects and side effects of lidocaine spray in neonatal populations during intubation. In the current study the mean blood pressure and heart rate of neonates in treatment group were reduced after intubation compared with their relevant figures before intubation but this reduction was not statistically significant.

Kelly and co-workers by a randomized trial of 30 newborns reported that decreases in transcutaneous $\mathrm{PO}$, increase the mean arterial blood pressure and intracranial pressure in infants receiving atropine, atropine plus pancuronium, or no premedication before intubation. Infants who were not premedicated experienced significantly more decreases in heart rate and demonstrated the lowest mean heart rate, compared with the other two groups. Pancuronium plus atropine was associated with less increase in intracranial pressure, heart rate, or systemic blood pressure in response to intubation (11). The difference between our results and Kelly study may be related to the different drugs used in these two studies.

Pathak and coworkers studied to determine whether lidocaine and/or alfentanil can effectively attenuate mean arterial pressure (MAP), heart rate (HR), and rate pressure product (RPP) associated with tracheal intubation. They found that alfentanil completely inhibited the hemodynamic effects of intubation but lidocaine had no effect on these variables (12). The results of this study which used the same drug and method as we did were in accordance with the results of the current study. Although there was a non significant reduction in the mean heart rate and blood pressure after intubation compared to before intubation; but a study with larger sample size is needed to reveal the possible effectiveness of lidocaine in the reduction of these factors.

Chaudhary et al. evaluated current practices for premedication use prior to elective intubation in tertiary neonatal units in the UK of which $90 \%$ reported a routine use of sedation prior to intubation and $82 \%$ a routine use 
of muscle relaxants. Morphine was the most commonly used sedative and suxamethonium was the most commonly used muscle relaxant. Approximately half of the units also used atropine during intubations. Seventy seven percent of units had a written policy for premedication and only ten percent of the units did not use any sedatives or muscle relaxants for elective intubations routinely (21). Wheeler and coworkers described the current approach to premedication in neonatal intensive care units (NICUs) in Australia and New Zealand and found that all tertiary NICUs and neonatal emergency transport services in Australia and New Zealand use premedication for elective intubation of neonates. Eighty percent of units had a written policy. There were 28 of 30 (93\%) units that used muscle relaxants, mostly suxamethonium (22).

Durrmeyer et al. described the frequency and nature of premeditations used prior to neonatal endotracheal intubation and found that the specific premedication rate was $56 \%$ and included mostly opioids (67\%) and midazolam (53\%). According to the recent guidance from the American Academy of Pediatrics, uses of premeditations could be classified as "preferred" (12\%), "acceptable" (18\%), "not recommended" (27\%), and "not described" (43\%) (23). These drug applications during neonatal intubation indicate a practical difficulty for kids' intubation particularly during neonatal period worldwide and our study was the first attempt in Iran, using lidocaine spray as a possible effective tool for this purpose. Using a safe drug with the least side effects prior to neonatal intubation can help practitioners to perform this procedure in neonates without any complication. According to the results of current study, application of lidocaine spray prior to intubation shortens the mean time of intubation and helps to maintain stability of oxygen saturation rate, heart rate and blood pressure during intubation. No side effects such as convulsion, arrhythmia, and decreased level of consciousness were observed during study in lidocaine group. The results of this study were inconsistent with those reported by Megan Brooks, who reported that oral viscous lidocaine $2 \%$ solution should not be used to treat infants and children with teething pain because of a serious risk (16). The current study used lidocaine medicine in spray form as much as one puff at the mucosal surface with the least absorption rate; however, Brooks report was about using this drug as a viscous solution form with higher dosage and by an oral path. The different types of drug applications and different dosages of this medicine may justify the inconsistency between Brooks report and the results of the present study.

As a conclusion, application of lidocaine spray can shorten the mean time of neonatal intubation significantly and reserves the vital signs such as blood pressure, heart rate and oxygen saturation rate in a stable status. Though the current study revealed some promising results in the application of lidocaine spray during neonatal intubation without any considerable side effects; how- ever, the current investigation could only be considered as a pilot study for further attempts in different locations with higher sample sizes and in different situations.

The limitations of the present study were lack of data related to serum levels of the drug and small sample size. A future study with higher sample size, to investigate the relationship between serum level of lidocaine and studied variables can increase the quality and preciseness of results detected in the current study.

\section{Acknowledgements}

We express our sincere thanks and gratitude to the NICU nurses of Mustafa Khomeini Hospital and Vice Chancellor for Researches and Technology of Ilam University of Medical Sciences for their valuable collaboration.

\section{Funding/Support}

Ilam University of Medical Sciences.

\section{References}

1. Carbajal R, Eble B, Anand KJ. Premedication for tracheal intubation in neonates: confusion or controversy? Semin Perinatol. 2007;31(5):309-17.

2. Marshall TA, Deeder R, Pai S, Berkowitz GP, Austin TL. Physiologic changes associated with endotracheal intubation in preterm in fants. Crit Care Med.1984;12(6):501-3.

3. Anand KI, Hickey PR. Pain and its effects in the human neonate and fetus. N Engl J Med.1987;317(21):1321-9.

4. Sarkar S, Schumacher RE, Baumgart S, Donn SM. Are newborns receiving premedication before elective intubation? J Perinatol. 2006;26(5):286-9.

5. Venkatesh V, Ponnusamy V, Anandaraj J, Chaudhary R, Malviya M, Clarke P, Arasu A, Curley A, et al. Endotracheal intubation in a neonatal population remains associated with a high risk of adverse events. Eur J Pediatr. 2011;170(2):223-7.

6. VanLooy JW, Schumacher RE, Bhatt-Mehta V. Efficacy of a premed ication algorithm for nonemergent intubation in a neonatal intensive care unit. Ann Pharmacother. 2008;42(7):947-55.

7. DeBoer SL, Peterson LV. Sedation for nonemergent neonatal intubation. Neonatal Netw. 2001;20(7):19-23.

8. Mosalli R, Shaiba L, Alfaleh K, Paes B. Premedication for neonatal intubation: Current practice in Saudi Arabia. Saudi J Anaesth. 2012;6(4):385-92.

9. Millar C, Bissonnette B. Awake intubation increases intracranial pressure without affecting cerebral blood flow velocity in infants. Can J Anaesth. 1994;41(4):281-7.

10. Friesen RH, Honda AT, Thieme RE. Changes in anterior fontanel pressure in preterm neonates during tracheal intubation. Anesth Analg.1987;66(9):874-8.

11. Kelly MA, Finer NN. Nasotracheal intubation in the neonate: physiologic responses and effects of atropine and pancuronium. JPediatr. 1984;105(2):303-9.

12. Pathak D, Slater RM, Ping SS, From RP. Effects of alfentanil and lidocaine on the hemodynamic responses to laryngoscopy and tracheal intubation. J Clin Anesth. 1990;2(2):81-5.

13. Anand KJ, International Evidence-Based Group for Neonatal P. Consensus statement for the prevention and management of pain in the newborn. Arch Pediatr Adolesc Med. 2001;155(2):173-80.

14. American Academy of Pediatrics Committee on F, Newborn, American Academy of Pediatrics Section on S, Canadian Paediatric Society F, Newborn C, Batton DG, Barrington KJ, Wallman $C$, et al. Prevention and management of pain in the neonate: an update. Pediatrics. 2006;118(5):2231-41.

15. Kumar P, Denson SE, Mancuso TJ, Committee on F, Newborn SOA Pain M. Premedication for nonemergency endotracheal intubation in the neonate. Pediatrics. 2010;125(3):608-15. 


\section{Mussavi Met al.}

16. Brooks M. Oral Lidocaine for teething pain may cause harm, FDA Says. 2014. Available from: http://www.medscape.com/viewarticle/827419.

17. Eyres RL, Bishop W, Oppenheim RC, Brown TC. Plasma lignocaine concentrations following topical laryngeal application. Anaesth Intensive Care.1983;11(1):23-6.

18. Veyckemans F, Matta A, Gribomont BF, Kestens-Servaye Y. A safe alternative to awake intubation in neonates. Acta Anaesthesiol Belg. 1985;36(3):143-50.

19. Eyres RL, Kidd J, Oppenheim R, Brown TC. Local anaesthetic plasma levels in children. Anaesth Intensive Care. 1978;6(3):243-7.

20. Pelton DA, Daly M, Cooper PD, Conn AW. Plasma lidocaine concentrations following topical aerosol application to the trachea and bronchi. Can Anaesth Soc J.1970;17(3):250-5.

21. Chaudhary R, Chonat S, Gowda H, Clarke P, Curley A. Use of premedication for intubation in tertiary neonatal units in the United Kingdom. Paediatr Anaesth. 2009;19(7):653-8.

22. Wheeler B, Broadbent R, Reith D. Premedication for neonatal intubation in Australia and New Zealand: a survey of current practice. J Paediatr Child Health. 2012;48(11):997-1000.

23. Durrmeyer X, Daoud P, Decobert F, Boileau P, Renolleau S, ZanaTaieb E, Saizou C, Lapillonne A, Granier M, Durand P, Lenclen R, Coursol A, Nicloux M, de Saint Blanquat L, Shankland R, Boelle PY, Carbajal R, et al. Premedication for neonatal endotracheal intubation: results from the epidemiology of procedural pain in neonates study. Pediatr Crit Care Med. 2013;14(4):e169-75. 